\title{
Pensée sociale et pratiques langagières de futurs enseignants de l'école primaire vaudoise : les savoirs construits lors du stage professionnel
}

\section{$\mathrm{R}$}

L'objet de cet article consiste à comprendre la nature des savoirs de référence construits par des futurs enseignants primaires vaudois lors de leur stage professionnel. L'approche retenue consiste à développer les notions de pensée sociale, mondes et pratiques langagières, dans le contexte de l'enseignement d'une géographie renouvelée. Diverses données ont été recueillies dans le cadre d'une recherche collaborative orientée par la conception, auprès de huit futurs enseignants de l'école primaire : des entretiens ante et post, des enregistrements de leçons ainsi que les supports de cours. Les analyses font apparaitre des discours contrastés portant sur l'objet

d'étude - l'alimentation -, l'importance des notions, et le rôle crucial joué par l'expérience des élèves. L'article se termine par une réflexion portant sur l'apport du dispositif de recherche collaborative ainsi que sur les notions d'alternance et de référence.

Mots-clés

Géographie, pensée sociale, pratiques langagières, savoirs de référence, stage professionnel

Abstract

The purpose of this article is to understand the nature of the reference knowledge built by future primary teachers (in the state of Vaud) during their traineeship. The approach is to develop the concepts of social thought, worlds and language practices in the context of the teaching of a renewed geography. Various data were collected as part of collaborative design-based research with eight future primary school teachers: ante and post

interviews, lesson recordings as well as course materials. The analysis reveal contrasting discourses about the object of study - nutrition - the importance of notions and the crucial role played by the students' experience. The article ends with a reflection on the contribution of the collaborative research system as well as the notions of alternation and reference.

\section{Keywords}

Geography, social thought, language practices, reference knowledge, traineeship

\section{Introduction}

La question des savoirs à enseigner dans les disciplines des sciences humaines et sociales est cruciale à plus d'un titre. En effet, ces disciplines - la géographie, l'histoire et l'éducation à la citoyenneté se trouvent aujourd'hui dans une situation où elles doivent cohabiter avec de nouveaux objets, comme l'éducation aux médias, l'éducation à la santé ou encore l'éducation en vue du développement durable. Selon Tutiaux-Guillon (2009), un tel enseignement se heurte aux logiques de la forme scolaire (Vincent, 1980, 1994), car il implique une « triple rupture » : une rupture épistémologique, une rupture avec les finalités et une rupture avec les pratiques. Sur un plan épistémologique, il s'agit de «faire de la place à l'incertitude, au désordre, à l'instabilité, à la pluralité des interprétations ", mais aussi « au futur » (TutiauxGuillon, 2009, p. 148). En outre, il s'agit de réfléchir à l'articulation entre les multiples dimensions prises en compte par ces Éducations $\grave{a} \ldots$.. les dimensions politiques et juridiques, sanitaires, économiques, sociales et démographiques, culturelles, religieuses, éthiques ou encore techniques -, et les outils de pensée disciplinaires - autrement dit les concepts centraux des disciplines, qui ne les recouvrent pas totalement. En effet, les questions qui se posent à nos sociétés ne sont pas disciplinaires et les solutions qui peuvent leur être apportées ne le sont pas non plus (Audigier, 2001). Autrement dit, il s'agit de dépasser les catégories disciplinaires sans pour autant les effacer, ce qui revient à envisager la contribution de chaque discipline au projet collectif, mais aussi de réfléchir en termes de hiérarchie et de positionnement réciproque (Vergnolle-Mainar, 2011). En effet, les différentes disciplines scolaires ne construisent pas les mêmes relations de l'individu à lui-même et au monde (Audigier, 2012b). 
Dans le cadre de cet article, nous partons du postulat selon lequel la géographie scolaire peut être rattachée, sur un plan épistémologique, aux sciences du monde social, autrement dit aux sciences socio-historiques (Passeron, 1991). Celles-ci permettent la catégorisation du réel et le découpage en domaine d'études - l'histoire, la sociologie, la géographie. Elles s'appuient sur l'enquête, le recueil, la construction de sources et la production de textes. En outre, elles s'intéressent aux actions humaines dans leur contexte et étudient particulièrement les acteurs, les processus de décision ainsi que le rôle joué par les intérêts divers, les opinions, les croyances ou les valeurs (Audigier, 2012a).

La deuxième rupture, mentionnée plus haut, renvoie aux finalités de ces disciplines : quelle place respective accorder aux finalités "patrimoniales et civiques", aux finalités "intellectuelles et critiques », ainsi qu’aux finalités « pratiques » (Audigier, 1995)? Ces trois catégories sont généralement mentionnées dans les plans d'études, mais la logique des compétences et de l'employabilité a tendance à favoriser la valeur d'utilité sociale. Dès lors, comment s'articulent les savoirs dont les élèves auront besoin et les actions qu'ils pourraient mener dans l'espace social? Comment prendre en compte leur expérience dans le processus d'enseignement?

En regardant de plus près, comment faut-il se positionner par rapport à des normes qui envahissent l'espace public et qui, souvent, dévalorisent les savoirs et prônent les bienfaits de l'expérience? ${ }^{1}$ Comment échapper à la « dictature du présent» (Audigier, 2012b) pour penser le futur? Comment faire preuve d' « interprétation inventive " (Citton, 2010) ${ }^{2}$ ? Que dire alors des approches d'éducation en vue du développement durable qui se limitent à prôner des éco-gestes ${ }^{3}$, relayant ainsi un discours social dominant?

De telles questions sont centrales et n'ont pas encore été toutes abordées dans les recherches en didactique, qui nont que peu développé d'outils permettant d'étudier cette nouvelle réalité.

Enfin, le troisième champ de « rupture » concerne les pratiques. Dans le contexte de l'école primaire, le projet éducatif visant à former de futurs citoyens est rendu particulièrement complexe pour plusieurs raisons. Il faut rappeler, tout d'abord, que les enseignants de cet ordre ne peuvent pas s'appuyer sur une formation académique en sciences sociales. Ils ont donc tendance à reproduire une géographie scolaire qu'ils ont connue, à savoir sur un enseignement fondé sur un "modèle dominant " (Audigier, 1987) - le cours magistral dialogué - qui ne prend généralement pas en compte les questions d'actualité, les valeurs, l'action et le social (Audigier et Tutiaux-Guillon, 2004), et qui s'appuie sur des documents présentés comme des substituts du réel (Tutiaux-Guillon, 2009). Il n'est pas inutile non plus de rappeler qu'à l'époque de cette recherche, dans les cantons romands, ces enseignants ne pouvaient pas encore tous travailler avec des moyens d'enseignement mettant en évidence les principales caractéristiques d'une géographie recomposée; ils devaient donc faire preuve d'une certaine créativité en inventant des tâches et des dispositifs adéquats ${ }^{4}$. En outre, comme l'ont montré des études récentes axées sur les pratiques à l'école primaire, le statut du maître généraliste enseignant sept à huit disciplines ne favoriserait pas l'accession à un rapport distancié au monde, ni la mise en œuvre d'une véritable interdisciplinarité (Philippot, 2009).

Dans la suite de cet article, nous développons les notions de pensée sociale, mondes et pratiques langagières, dans le contexte de l'enseignement d'une géographie renouvelée. Puis, après une brève présentation de la problématique et du contexte de la recherche, nous mentionnons quelques résultats, à savoir trois discours contrastés portant sur l'objet d'étude - l'alimentation -, l'importance des notions, 
et le rôle crucial joué par l'expérience des élèves. Nous terminerons par une réflexion portant sur l'apport du dispositif de recherche collaborative, ainsi que sur les notions d'alternance et de référence.

\section{Pensée sociale, mondes et pratiques langagières}

L'objet de cet article consiste à comprendre la nature des savoirs de référence construits lors du stage professionnel. Cela consiste à étudier l'expression et le développement de la pensée sociale des futurs enseignants primaires vaudois, sur le thème de l'alimentation, à travers le prisme de la géographie scolaire. Par " pensée sociale ", nous entendons à la fois la manière dont chacune et chacun construit une pensée de la vie sociale, et ce qu'elle ou il utilise pour penser et communiquer avec les autres, que ce soit par oral ou par écrit (Audigier et Haeberli, 2004). Deux notions méritent donc d'être présentées d'emblée pour préciser notre propos : les notions de "monde » et de " pratiques langagières ».

Pour nous, l'enjeu central de l'enseignement des disciplines de sciences sociales consiste à permettre aux élèves de s'imaginer des mondes différents des leurs, qu'ils soient passés, présents ou futurs. Nous retiendrons l'idée de Goodman (1978/1992) selon laquelle il existe une multitude de mondes qui se construisent, se combinent ou se découvrent au fur et à mesure que la connaissance progresse. Ces mondes se créent principalement par le langage qui fonctionne comme un "multiplicateur » et un « mélangeur» de mondes (François, 1993, p. 116).

Dès lors, l'étude fine des manières dont les mondes se construisent - autrement dit, l'étude des pratiques langagières (Bautier, 1995) -, nous paraît tout aussi importante, si ce n'est plus, que la description des mondes que des futurs enseignants construisent. Par pratiques langagières, nous faisons référence au fonctionnement du langage en contexte et à la production de sens. Plus généralement, ces pratiques interrogent le rapport du sujet au langage et à la situation dans laquelle il est engagé (Reuter, 2007).

Il convient aussi de rappeler, avec Bruner (1986), que le langage n'est jamais neutre. Il impose un point de vue sur le monde auquel il se réfère. Ainsi, l'École a pour fonction d'introduire les élèves dans une culture, c'est-à-dire dans des langages spécifiques et donc dans des «mondes construits». Mais le langage scolaire a aussi pour fonction de décrire « la manière dont l'esprit est utilisé par rapport à ce monde » (Bruner, 1986, p. 147). Il n'y a donc pas de monde sans langage et il n'y a pas de pensée sociale sans mondes et sans langage.

Si notre choix s'est porté sur le thème de l'alimentation, c'est parce qu'il nous paraît potentiellement intéressant pour mettre en lumière trois séries de phénomènes : les enjeux sociaux d'aujourd'hui et de demain, les enjeux de la recomposition disciplinaire à l'école primaire (mentionnés en introduction) et les spécificités de la pensée sociale, qui, comme nous le verrons, articule des savoirs de natures différentes, y compris des savoirs d'expérience, même chez les jeunes élèves. Enfin, l'articulation entre le proche et le lointain - certains aliments sont importés, d'autres pas -, mais aussi les dimensions d'identité et d'altérité intrinsèquement liées à l'alimentation en font un thème particulièrement riche à étudier.

Compte tenu de ces éléments théoriques, nous retiendrons, pour la suite de cet article, les deux questions de recherche suivantes : quels savoirs de futurs enseignants du primaire enseignent-ils autour du thème de l'alimentation, dans le cadre de la géographie scolaire? Quelle(s) modalité(s) de formation des futurs enseignants primaires faut-il privilégier? 


\section{Contexte et méthodologie de la recherche}

La recherche empirique dont nous allons faire état s'inscrit dans le modèle de la recherche collaborative orientée par la conception (Sanchez, 2014). Cela signifie que les différents partenaires - futurs enseignants et formateur - collaborent par le partage de praxéologies, autrement dit, par l'élaboration d'un discours commun sur la pratique. La particularité de cette recherche, toutefois, est liée au fait qu'elle fait suite à deux modules de formation à la didactique des sciences humaines. Le premier, intitulé «Didactique des sciences humaines 1 », met l'accent sur des savoirs disciplinaires de base (en histoire, en géographie, en histoire et sciences des religions), ainsi que sur la problématisation, la conceptualisation et l'usage des outils spécifiques à ces disciplines à l'école. Le deuxième module, "Didactique des sciences humaines 2 ", aborde trois contenus spécifiques: les recompositions disciplinaires induites par l'émergence des « Éducations à... » et des questions sociales vives (Legardez et Simonneaux, 2006); la différenciation de l'enseignement dans nos disciplines et l'intégration des nouvelles technologies de l'information et de la communication ${ }^{6}$. C'est au cours de cette deuxième année de formation que les futurs enseignants du primaire ont été sollicités pour participer à cette recherche.

Au total, 164 futurs enseignants formés à la HEP Vaud ont participé à une enquête quantitative (par questionnaire), à la fin de leur deuxième année de formation. Seuls huit d'entre eux ont participé à l'enquête qualitative, qui consistait à mettre en œuvre une démarche d'enseignement-apprentissage dans leur classe de stage. Des entretiens semi-directifs réalisés avant et après leurs enseignements ont permis d'obtenir des précisions quant aux choix didactiques et aux apprentissages réalisés. L'entretien ante comprenait cinq temps : les logiques de préparation, les situations didactiques, les supports, les savoirs et leur articulation, les difficultés envisagées et le rôle de l'enseignant. Quant à l'entretien post, il comptait trois temps : autour de ce qui s'est passé en classe, autour des apprentissages des élèves et autour de l'apprentissage de l'enseignant. Précisons enfin que cinq étudiants ont par ailleurs réalisé leur mémoire professionnel dans le cadre de cette recherche collaborative (figure 1).

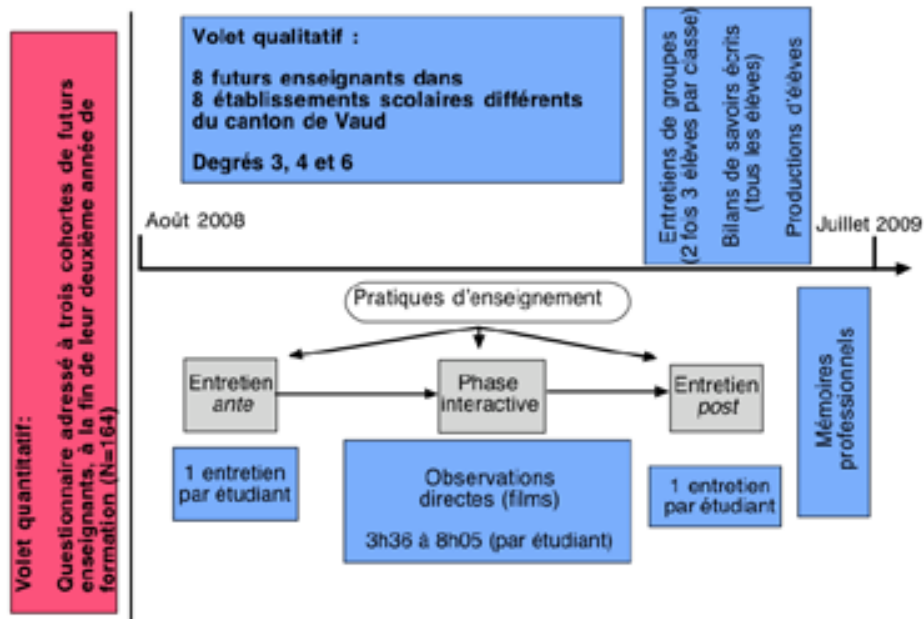

Figure 1

Le dispositif de production des données. 
Dans le cadre de ce texte, nous ne mentionnerons que quelques résultats issus de l'analyse qualitative. Afin d'analyser nos données, nous avons construit un modèle à partir de diverses disciplines des sciences sociales, et plus particulièrement à partir de la notion de récit, qui a été travaillée par Bruner (2002) et Ricœur (1983-1985). Cette notion constitue un fil conducteur permettant de faire la "synthèse de l'hétérogène " (Ricœur, 1983-1985) et de traduire les expériences humaines. Cela se produit notamment par l'intermédiaire d'une « mise en intrigue » qui accentue certains aspects et en occulte d'autres, en vertu des processus d'objectivation par sélection et de focalisation, bien décrits par les psychologues sociaux.

Comme le rappelle aussi Haeberli (2009), le récit « se présente comme une manière particulière de construire des mondes mettant en œuvre un ensemble d'activités complexes du ressort du langage et des autres systèmes symboliques » (p. 122). Cette complexité peut s'appréhender, comme nous l'avons vu, à l'aide des « genres discursifs » qui évoluent et donnent naissance à des « variations perpétuelles de mondes » (François, 1993).

Étant par ailleurs considéré comme «le registre fondamental de l'être-au-monde des individus » (Lussault, 2007, p. 220), le récit permet de convertir «l'intériorité en extériorité ». Cela signifie que des valeurs, des savoirs, des opinions, des intentions sont ainsi mises à jour, de sorte que l'on peut parler avec Lussault de « récit d'action ». Ce récit d'action est d'autant plus nécessaire aujourd'hui dans le cadre du projet global de la formation du citoyen.

Les récits d'action dont nous allons faire état sont issus des discours et des pratiques mises en œuvre par les étudiants. Ils seront décrits sur la base de cinq dimensions ayant émergé de notre cadre théorique : les savoirs, les formes langagières et processus de pensée sociale, les registres et disciplines du monde social, les activités formelles de l'enseignant et les auxiliaires utilisés. Une telle analyse par catégories conceptualisantes (Paillé et Mucchielli, 2003) a été complétée par une analyse textuelle, réalisée avec le logiciel Alceste, et par une analyse dialogique des interactions, qui permet de tirer parti de la richesse des discours produits tout en mettant en évidence l'instabilité et l'hétérogénéité des représentations sociales (Salazar Orvig et Grossen, 2004).

Les connaissances identifiées chez les futurs enseignants seront présentées en trois temps dans la suite de ce texte : nous examinerons successivement l'objet à enseigner, le rôle de la géographie scolaire et enfin la conception du savoir.

\section{Trois discours contrastés et trois « mondes »}

En ce qui concerne l'objet à enseigner, nous avons pu mettre en évidence trois discours contrastés et trois «mondes ». Ceux-ci apparaissent sur le schéma d'analyse factorielle des correspondances en coordonnées fourni par le logiciel Alceste (figure 2). Rappelons, pour les non-initiés, que le logiciel dégage, à partir d'un large corpus (en l'occurrence des entretiens semi-directifs), des classes de discours, autrement dit, des séries de termes utilisés ensemble ${ }^{7}$. Nous présentons ci-dessous les trois classes les plus spécifiques en respectant l'ordre de partition du corpus. Chaque classe comprend généralement deux sous-ensembles de termes. 
La première classe de discours se présente comme la plus spécifique, car elle est issue de la première partition du corpus. Deux mots sont particulièrement associés à cette classe de discours : " produit » (le khi-deux est de 368) et "terroir " (le khi-deux est de 238) ${ }^{8}$. Dans ce même sous-ensemble, on trouve des exemples de produits (le «vin », le «fromage »), des indicateurs de qualité ( AOC », «logo », "typique»), mais aussi des termes qui rendent compte de la dimension spatiale de l'alimentation (« région », « endroit », « lieu » ou encore « Lavaux »).

Un deuxième sous-ensemble de termes met en évidence des habitudes de consommation qui favorisent les produits locaux. Sont mentionnés des produits ( "tomate », « lait »), des références spatiales ou des lieux de consommation ("Suisse ", " local », «France », " magasin », " supermarché »), ainsi que des actions (« acheter », « connaître », « choisir », « venir »).

La deuxième classe de discours regroupe deux sous-ensembles de formes lexicales. Le premier est articulé autour de deux mots dont la valeur statistique d'association est très forte : "céréales " (le khideux est de 251) et «blé » (le khi-deux est de 159). Les autres mots renvoient à des savoirs factuels : " exister ", " parcours ", " cultiver ", " origine ", " historique ». On trouve aussi des adverbes qui témoignent du souci de faire référence à l'actualité («actuellement»), mais qui traduisent aussi une certaine hésitation quant aux contenus à aborder (« éventuellement»).

Le deuxième sous-ensemble de mots aborde le thème des "allergies ", et plus particulièrement, les allergies au "gluten ", dans une perspective d'éducation à la "santé ». Ainsi, plusieurs actions sont évoquées : «manipuler ", " reconnaître ", " comporter ", «toucher ». Même si la valeur du khi-deux est plus faible, il est intéressant de mentionner le «futur » et le registre du quotidien, évoqué par des formes comme « caddie », « famille », " parent».

La troisième classe de discours regroupe trois sous-ensembles de formes. Le premier est articulé autour du mot «aliment » (le khi-deux est de 108) et de l'idée de «transformation ». Cette dernière renvoie à une «histoire », comme l'indique l'unité de contexte élémentaire la plus caractéristique de cette classe.

[L'enjeu, c'est] donc vraiment se rendre compte que c'est pas un simple pot de yogourt, que tout... qu'il y a une histoire de l'aliment qui est derrière... cet aliment. (u.c.e. 142)

Le mot «brut », fortement associé à cette classe, appartient aussi à ce sous-ensemble.

Le deuxième sous-ensemble de termes aborde le «voyage » des aliments et mentionne les exemples du " yogourt » et du « chocolat ». Enfin, le troisième sous-ensemble de mots fait référence à l'« image », au " matériel », qui doit être « concret », afin de favoriser des habitudes de " consomm'acteurs ».

L'analyse des pratiques a confirmé l'hypothèse selon laquelle des récits ont été construits autour de ces trois classes de discours. Ces trois classes prennent donc la forme de trois « intrigues types»:

- Lavaux ${ }^{9}$, les produits du terroir et les appellations d'origine contrôlée (AOC);

- les céréales et les allergies alimentaires;

- la transformation et le voyage des aliments. 


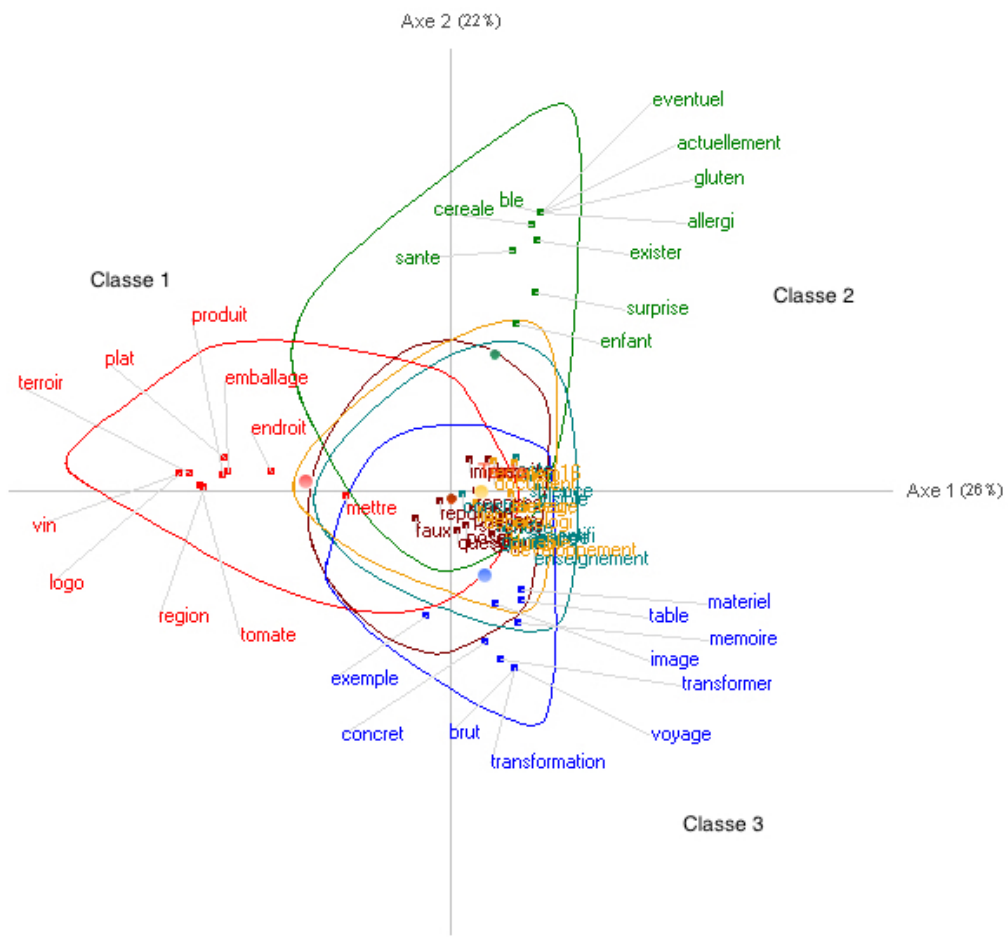

Figure 2

\section{L'analyse factorielle des correspondances en coordonnées.}

\section{Une géographie scolaire centrée sur des notions et au service d'un projet plus global}

Les entretiens réalisés ont mis en évidence une conception de la géographie centrée sur des notions que l'on utilise «dans la vie de tous les jours ». Ces notions ne sont donc pas présentées comme des constructions disciplinaires, mais plutôt comme des savoirs utiles pour comprendre le monde. Ce sont des expressions ou mots comme "agriculture biologique ", "produit transformé ", " traçabilité » ou encore « consomm'acteur » qui ont donc été choisis en priorité et qui sont présentés au moyen de définitions.

Ces savoirs sont assimilables à des « fondations » qu'il s'agirait de construire dans un premier temps. Voici comment Daniel, un des enseignants du corpus, s'exprime à ce sujet :

Extrait 1 : Daniel_entretien ante

Je dirais que la notion, c'est... pour moi c'est ce qui est en bas de la pyramide. Dans le sens que c'est la base sur laquelle elle doit être construite. On... on peut essayer de construire une méthode de travail. On peut essayer de construire des... des compétences sans faire ça, mais si on n'a pas un minimum de connaissances sur le thème, on est perdu. Et ça euh... bon, je sais qu'il y a certaines... beaucoup de théories qui s'affrontent là autour, euh... [...] Il faut construire sur quelque chose (inaudible) ... sur des fondations... 
Mais ces notions sont au service d'un projet plus global, celui qui consiste à comprendre les enjeux planétaires actuels et les "problèmes rencontrés ».

Extrait 2:Daniel_entretien ante

Donc... je pensais euh... les confronter la prochaine fois euh... aux problèmes de... de faim dans le monde et en particulier aux problèmes liés au fait que certains pays exportent des denrées qui sont consommées chez nous par exemple. Et qui ont des... des problèmes de faim dans leur pays.

Dès lors, les notions abordées sont au service du raisonnement et de la perspective citoyenne :

Extrait 3 : Daniel_entretien post

[...] les notions que j’ai apportées euh... bon, je leur ai jamais vraiment demandé de les apprendre par cœur. Je leur ai demandé d'être capables de les réexpliquer et d'être capables d'en faire quelque chose dans... dans une situation bien... bien précise. Donc d'être capables de les utiliser. Ce qui à mon avis est plus compliqué et beaucoup plus exigeant que d'apprendre par cœur.

\section{L'expérience des élèves et son influence sur la conception des savoirs}

Plusieurs enseignants ont été déstabilisés suite à leurs interventions en classe. En effet, certaines réactions d'élèves leur ont permis de prendre de la distance par rapport aux savoirs qu'ils enseignaient. C'est par exemple le cas d'Aloïs qui remet en question sa conception du développement durable : il prend ainsi conscience de la complexité des actions individuelles, lesquelles ne peuvent se réduire à de simples injonctions :

Extrait 4 : Aloï_entretien post

[...] Par rapport au développement durable, après coup j’ai vu que c'était peut-être dans une extrême parce qu'à la fin j'ai dû vraiment avertir que c'était vraiment une généralité. Que c'était pas soit ça, soit ça. Qu'il fallait aussi euh... ben par rapport euh aux facteurs économiques... j’en avais une dans ma classe, elle regardait à chaque fois son jus d'orange et pis euh... elle disait à chaque fois à sa maman mais euh... pis la maman c'était l'enseignante euh de la classe à côté. Pis elle a dit mais c'est... c'est insupportable à la maison. Et pis euh... Ben qu'il fallait pas euh... prendre du jus d'orange parce qu'il venait... il venait du Brésil ou euh... Ou bien par rapport à d'autres aliments mais qui... à la fin euh... ben cette... cette fille après elle faisait... cette élève elle faisait une généralité de tout ce quelle voyait à la maison, des produits... de euh... où ils viennent, comment... Ben en fait elle avait pas fait... enfin... moi j'ai pas insisté justement sur le fait que le facteur environnemental, c'est une chose. Le facteur social, c'est une chose. Mais que le facteur économique ma foi, il influence euh... ces... ces deux pôles.

Pour Aloïs, les savoirs à enseigner font l'objet d'un questionnement permanent tout au long de la démarche menée en classe. Il en vient même à visionner un film documentaire produit par des climatosceptiques. Cette expérience l'amènera à revoir sa conception du savoir et à mettre l'accent sur le « tri des informations médiatiques». 


\section{Extrait 5 : Aloï_entretien post}

Ben justement après coup, ben j'avais fini la séquence de... de $\mathrm{CE}$ en classe tout. Et pis durant le week-end j'ai vu un reportage sur euh... enfin l'arnaque du réchauffement de la planète. Je sais pas si vous l'avez vu? [...] Et pis après ce film... enfin après tous ces épisodes... pis j'étais là mais... j'avais de la peine avec moi-même parce que je me disais mais est-ce que j'ai transmis quelque chose de bien ou de valable ou est-ce que j'ai appris à mes élèves à... à développer une logique, mais une logique que... une fausse logique d'un côté. Donc ce que j'aurais mieux fait, je pense, c'est de... ouais de leur apprendre à raisonner mais pas de manière... avec une seule grille d'analyse je pense. [...] C'est ce que j'aurais dû peut-être plus mettre l'accent... le développement durable, c'est une chose... mais... bon on pourrait mettre comme des étages. Il y a le développement durable. Mais en dessous il y a différentes euh palettes qui se cachent. Par exemple sur chaque euh... facteur on aurait différents documents de bases de références. Et pis c'est justement là-dessus qu'il faudrait euh... plus travailler. Parce que là on... je pense c'est un travail un peu en surface quoi. Donc peut-être qu'à cet âge-là c'est bien de travailler en surface pour mettre des bases. Mais je sais pas si ces bases deviennent doctrines ou... ou autre quoi. [...] Donc est-ce qu'il faudrait pas faire de temps en temps un cours de CE sur le tri des informations médiatiques presque? [...]... Je pense... si je refais le... euh le sujet, je pense qu'au préalable, il y aura beaucoup plus un travail sur euh... qu'est-ce qui est vrai, qu'est-ce qui est pas vrai.

D'autres futurs enseignants de notre échantillon ont été amenés à revisiter leur conception du savoir. C'est par exemple le cas de Sylvie, qui, lors de l'entretien ante n'insistait que sur des savoirs factuels. Or, dans l'entretien post, elle se montre capable d'articuler les savoirs travaillés en les rangeant dans des catégories.

\section{Extrait 6: Sylvie_entretien post}

J'ai pu passablement euh... toucher mon fil rouge, les céréales et leur faire voir dans différents... euh de différents points de vue, de l'évolution historique, géographique, sociale, etc... [...] Je souhaitais parler euh... donc de de l'alimentation, de partir de l'alimentation pour suivre le fil rouge des céréales, euh, en parler de façon justement euh historique, dans l'environnement pourquoi euh... pourquoi est-ce qu'il y a des problèmes économiques actuellement, pourquoi est-ce qu'on a besoin du blé, où est-ce qu'il se trouve.

Après l'expérience menée en classe, Sylvie s'intéresse en outre davantage aux facteurs expliquant les différentes pratiques alimentaires (et non plus seulement à la localisation des céréales).

\section{Extrait 7: Sylvie_entretien post}

Pour moi, cétait euh... à l'aide d'outils géographiques, des cartes, voilà... [...] et ensuite de voir qu'il y a différents continents. Sur ces continents, différentes cultures euh... cultures au sens culturel et différentes habitudes justement alimentaires dues au sol, dues à... aux habitudes, dues au climat. Donc ça c'est toutes des thématiques pour moi géographiques... Donc c'était l'origine et le déplacement de... des céréales au départ de la Mésopotamie vers l'Europe, où c'était quand même tout ce qui est blé. On parlait du blé, là en l'occurrence, vers l'Europe. 


\section{Pause}

À ce stade, nous pouvons revenir sur le concept de pratiques langagières en tentant de l'approfondir. En effet, nous constatons que ces pratiques englobent à la fois les dimensions culturelles, sociales et langagières singulières - propres au sujet qui les produit - et partagées - soit propres au groupe qui les reconnaît et en a élaboré les formes. Ainsi, le langage, comme l'a montré Bautier (2001), permet à la fois de penser le monde et de s'inscrire dans une société.

Mais les discours des futurs enseignants vaudois étudiés renvoient aussi à des questions socialement vives (Legardez et Simonneaux, 2006). En voici quelques-unes : quels facteurs du développement durable privilégie-t-on? Pourquoi l'accès aux denrées alimentaires n'est-il pas équitable pour tous? Comment valoriser certains territoires? Comment expliquer les habitudes alimentaires?

Ces mêmes discours traduisent en outre une conception d'une géographie scolaire renouvelée. En effet, celle-ci intègre des finalités transdisciplinaires, autrement dit, des demandes sociales qui renvoient à l'éducation à la santé, à l'éducation aux médias ou à la formation du citoyen.

Enfin, nous constatons que le rapport au savoir des futurs enseignants se modifie avec une prise en compte de la complexité du monde : aucune solution n'est évidente, ce qui est vrai aujourd'hui ne le sera peut-être pas demain. Les savoirs doivent être en permanence remis en question de manière critique.

\section{La recherche collaborative orientée par la conception : une modalité de formation?}

Mais les exemples présentés ci-dessus illustrent aussi le fait que les savoirs construits lors du stage peuvent constituer une référence solide pour l'étudiant. Toutefois, il est nécessaire qu'un accompagnement soit mis en place. En effet, il est nécessaire d'offrir un espace de réflexion et d'échanges, afin de susciter un questionnement régulier sur les choix et les pratiques des futurs enseignants. Un tel dispositif permet aussi au formateur de valider des savoirs que l'étudiant s'approprie et qui font aussi référence dans les champs scientifique ou didactique.

La recherche collaborative orientée par la conception est donc une modalité qui nous paraît particulièrement féconde, car elle met en relations plusieurs étudiants et un chercheur/formateur qui partagent les mêmes interrogations et qui sont donc amenés à trouver collectivement des éléments de réponse. Mais encore faut-il garder une trace de ce processus de formation. Cela peut être fait dans un journal de bord ou dans un texte résumant le questionnement et la démarche menée. C'est cette dernière modalité qui a été retenue par cinq de nos étudiants (sur les huit ayant participé à notre recherche collaborative).

Le passage par l'écrit est une étape importante dans le processus de construction des apprentissages. En effet, l'écriture permet d'envisager ce qui a été dit ou pensé d'un œil nouveau. Elle réorganise l'appréhension du monde et favorise l'émergence et l'utilisation de concepts nouveaux. Elle permet donc d'entrer dans un nouvel univers en opérant une décontextualisation, comme l'ont mentionné plusieurs auteurs (Brossard, 2001; Goody, 1979; Olson, 1998).

87 - Formation et profession 25(1), 2017 
Les étudiants qui ont réalisé un mémoire professionnel ont donc tiré un maximum de bénéfices de cette recherche collaborative. En effet, leurs représentations ont évolué de manière plus marquée que les étudiants n'ayant pas réalisé de mémoire professionnel.

Ce dernier constat nous amène à envisager la recherche collaborative comme une modalité à part entière de la formation. Il reste toutefois à disposer d'un corps enseignant suffisamment important pour pouvoir encadrer des petits groupes d'étudiants. C'est peut-être ce dernier point qui sera le plus difficile à mettre en œuvre, à l'heure où les étudiants en formation sont de plus en plus nombreux.

\section{Conclusion}

En guise de conclusion, nous souhaitons prolonger la discussion sur la base de deux notions qui nous paraissent particulièrement fécondes : les notions d'alternance et de référence.

Pour ce qui est du modèle d'alternance mis en œuvre dans le cadre de cette recherche, il s'agit d'un modèle intégrateur par étapes (Vanhulle, Merhan et Ronveaux, 2007), puisque des phases de stage alternent avec des moments de réflexion, que ce soit une discussion autour d'une préparation de leçon, un entretien portant sur la justification des choix ou, de manière plus formelle, une soutenance de mémoire professionnel.

À chacune de ces étapes se construisent de nouveaux savoirs qui font référence pour les étudiants. Mais ces savoirs de référence sont aussi utiles pour le formateur qui, lors de cours et séminaires ultérieurs, peut réinjecter des éléments de pratiques d'anciens étudiants. C'est une manière d'accorder de l'importance aux difficultés rencontrées par les étudiants préscolaires et primaires, autrement dit, une manière dêtre empathique. Mais c'est aussi l'occasion de pallier la diminution des crédits de formation, le fait que certains étudiants ne suivent que peu de cours en didactique des sciences humaines et sociales ${ }^{10}$, et, $a$ fortiori, qu'ils ne réalisent de loin pas tous un mémoire professionnel dans ces disciplines.

\section{Notes}

1 À titre d'exemple, on pourrait citer Larry Sanger, l'un des fondateurs de Wikipédia, qui s'inquiète d'une nouvelle forme de dénigrement du savoir qu'il qualifie d'anti-intellectualism, ou encore certaines lectures de l'œuvre de Rousseau, qui opposent l'expérience aux savoirs (voir par exemple l'exposition organisée à Genève dans le cadre du Salon international du livre et de la presse, du 25 au 29 avril 2012).

2 Pour concevoir l'éducation de cette manière, Citton (2010, p. 121-126) propose sept « déplacements » : «apprendre à interpréter l'information plutôt que l'emmagasiner ", " partager le geste d'interprétation inventrice dans la présence interactive, plutôt que communiquer des contenus figés », " former des interprètes généralistes plutôt que des savants spécialisés ", " dynamiser les disciplines grâce à la créativité des sous-cultures minoritaires », " concevoir l'autoformation comme construisant des capacités communes de partage », " apprendre à intégrer l'argumentation logique au sein d'une vue plus large et critique des pratiques communicationnelles ", « reconfigurer l'université autour d'un grand axe mettant en tension les disciplines scientifiques avec l'indiscipline propre aux Humanités ».

3 À l'origine utilisé pour désigner les gestes écologiques du citoyen - trier ses déchets, utiliser des ampoules économiques, isoler les murs de sa maison -, le terme est aujourd'hui utilisé dans l'espace social avec une portée plus générale : il inclut, par exemple, la consommation de produits locaux, biologiques ou éthiques.

4 Dans la partie francophone de la Suisse (Genève, Vaud, Neuchâtel, Fribourg, Jura, Valais, une partie du canton de Berne), les manuels des degrés 7 et $8 \mathrm{H}$, par exemple, n'ont été livrés qu'à la rentrée d'août 2016. 
5 Par « interdisciplinarité », nous entendons une démarche d'enseignement qui s'appuie sur des outils et des modes de pensée propres à des disciplines (Fourez, 2002; Resweber, 1981).

6 Il est à noter que le séminaire relatif à la didactique de la géographie («Différencier en géographie ») a été centré sur le thème de l'alimentation.

7 Pour être très précis, il faudrait dire que l'arbre de la classification ascendante de chaque classe permet d'identifier les relations locales entre les formes d'une même classe. En effet, il met en évidence des paquets d'agrégation de formes ainsi que le khi-deux de chaque forme.

8 Nous ne mentionnons la valeur du khi-deux dans le texte que lorsque celle-ci est particulièrement élevée (autrement dit supérieure à 100) ou lorsqu'il s'agit de montrer une hiérarchie.

9 Il s'agit d'une région située au bord du lac Léman et inscrite au patrimoine mondial de l'UNESCO en 2008.

10 C'est notamment le cas des étudiants qui se destinent à l'enseignement dans les degrés 1 à 4 Harmos.

\section{Références}

Audigier, F. (1987). Histoire et géographie. Dans J. Colomb (dir.), Les enseignements en CM2 et en $6^{i m e}$. Ruptures et continuités (p. 45-111). Paris : INRP.

Audigier, F. (1995). Histoire et géographie : des savoirs scolaires en question entre les définitions officielles et les constructions des élèves. Spirale - Revue de recherches en éducation, (15), 61-89. Repéré à https://spirale-edu-revue.fr/IMG/pdf/3 AUDIGIER Spi15.pdf

Audigier, F. (2001). Le monde n'est pas disciplinaire, les élèves non plus, et les connaissances?. Dans G. Baillat et J.-P. Renart (dir.), Interdisciplinarité, polyvalence et formation professionnelle en IUFM (p. 43-59). Paris : CNDP et CRDP de Champagne-Ardenne.

Audigier, F. (2012a). Les Éducations à... et la formation au monde social. Recherches en didactiques, (14), 47-64.

Audigier, F. (2012b). Les Éducations à... Quels significations et enjeux théoriques et pratiques? Esquisse d'une analyse. Recherches en didactiques, (13), 25-38.

Audigier, F. et Haeberli, P. (2004, octobre). Des élèves, des images, de l'histoire, de la géographie, de la citoyenneté. Texte présenté au Colloque "Journée d'étude des didactiques de l'histoire et de la géographie », IUFM de Caen. Repéré à http://ecehg.ens-lyon.fr/ECEHG/colloquehgec/2004\%20Caen/jed2004audigier haeberli.pdf

Audigier, F. et Tutiaux-Guillon, N. (2004). Regards sur l'histoire, la géographie et l'éducation civique à l'école élémentaire. Paris : INRP.

Bautier, E. (1995). Pratiques langagières, pratiques sociales. De la sociolinguistique à la sociologie du langage. Paris : L'Harmattan.

Bautier, E. (2001). Pratiques langagières et scolarisation. Revue française de pédagogie, (137), 117-161. Repéré à http://ife.ens-lyon.fr/publications/edition-electronique/revue-francaise-de-pedagogie/INRP RF137 11.pdf

Brossard, M. (2001). Approche socio-historique des situations d'apprentissage de l'écrit. Dans M. Brossard et J. E. Fijalkow (dir.), Apprendre à l'école : perspectives piagétiennes et vygotskiennes (p. 37-50). Bordeaux : Presses universitaires de Bordeaux.

Bruner, J. (1986). Culture et modes de pensée. L'esprit humain dans ses œuvres. Paris : Retz.

Bruner, J. (2002). Pourquoi nous racontons-nous des histoires?. Paris : Retz.

Citton, Y. (2010). L'avenir des Humanités. Économie de la connaissance ou cultures de l'interprétation?. Paris: La Découverte.

Fourez, G. (2002). Approches didactiques de l’interdisciplinarité. Bruxelles : De Boeck.

François, F. (1993). Pratiques de l'oral. Dialogue, jeu et variations des figures du sens. Paris : Nathan.

Goodman, N. (1978/1992). Manières de faire des mondes. Paris : Gallimard.

Goody, J. (1979). La raison graphique. La domestication de la pensée sauvage. Paris : Éditions de Minuit. 
Haeberli, P. (2009). Contribution à l'étude d'une compétence dans le domaine de la citoyenneté. L'exemple de la justice dans les conseils de classe de l'école primaire genevoise (Thèse de doctorat, Université de Genève). Repéré à https://archive-ouverte.unige.ch/unige: 13237

Legardez, A. et Simonneaux, L. (dir.) (2006). L'école à l'épreuve de l'actualité. Enseigner les questions vives. Paris : ESF.

Lussault, M. (2007). L'Homme spatial. Paris : Seuil.

Olson, D. R. (1998). L'Univers de l'écrit : comment la culture écrite donne forme à la pensée. Paris : Retz.

Paillé, P. et Mucchielli, A. (2003). L'analyse qualitative en sciences humaines et sociales. Paris : Armand Colin.

Passeron, J.-C. (1991). Le raisonnement sociologique. Paris : Albin Michel.

Philippot, T. (2009, novembre). Les enseignants du primaire en France et l'interdisciplinarité : entre adhésion et difficile mise en cuvre. Texte présenté au Colloque international des didactiques de l'histoire, de la géographie et de l'éducation à la citoyenneté « Curriculums en mouvement », HEP Lausanne.

Reuter, Y. (2007). Dictionnaire des concepts fondamentaux des didactiques. Bruxelles : De Boeck.

Resweber, J.-P. (1981). La méthode interdisciplinaire. Paris : PUF.

Ricœur, P. (1983-1985). Temps et récit. Paris : Seuil.

Salazar Orvig, A. et Grossen, M. (2004). Représentations sociales et analyse du discours produit dans des focus groups : un point de vue dialogique. Bulletin de psychologie, 471(3), 263-272.

Sanchez, E. (2014). Recherche collaborative orientée par la conception : un paradigme méthodologique pour prendre en compte la complexité des situations d'enseignement-apprentissage. Éducation et didactique, 9(2), 73-94. http://dx.doi.org/10.4000/educationdidactique. 2288

Tutiaux-Guillon, N. (2009). Histoire-géographie et éducation au développement durable : entre modèle disciplinaire et nouvelles exigences. Dans F. Grumiaux et P. Matagne (dir.), Le développement durable sous le regard des sciences et de l'bistoire (vol. 1, p. 151-165). Paris : L'Harmattan.

Vanhulle, S., Merhan, F. et Ronveaux, C. (2007). Introduction. Du principe d'alternance aux alternances en formation des adultes et des enseignants : un état de la question. Dans F. Merhan, C. Ronveaux et S. Vanhulle (dir.), Alternances en formation (p. 7-45). Bruxelles : de Boeck. http://dx.doi.org/10.3917/dbu.merha.2007.01.0007

Vergnolle-Mainar, C. (2011). La géographie dans l'enseignement. Une discipline en dialogue. Rennes : Presses universitaires de Rennes.

Vincent, G. (1980). L'école primaire française. Lyon : Presses universitaires de Lyon.

Vincent, G. (dir.). (1994). L'éducation prisonnière de la forme scolaire. Scolarisation et socialisation dans les sociétés industrielles. Lyon : Presses universitaires de Lyon.

\section{Pour citer cet article}

Pache, A. (2017). Pensée sociale et pratiques langagières de futurs enseignants de l'école primaire vaudoise : les savoirs construits lors du stage professionnel. Formation et profession, 25(1), 78-90. http://dx.doi.org/10.18162/fp.2017.391 